\title{
Interact Through Your Data: Collective Immersive Experience Design for Indoor Exercises
}

\author{
$\mathrm{Xu}$ Lin, Linkai Tao, Bin Yu, Yongyan Guo, and Jun Hu${ }^{(\bowtie)}$ \\ Eindhoven University of Technology, \\ Den Dolech 2, 5612AZ Eindhoven, Netherlands \\ \{X. Lin, L. Tao, B. Yu, Y. Guo, J. Hu\}@tue.nl
}

\begin{abstract}
This paper presents an explorative design for improving indoor exercising experience, through real-time data visualization and social connection in an immersive environment. A prototype was designed and implemented based on the review of related research on cognitive and mental models. Facing existing design challenges, the project aims to find potential opportunities for future indoor exercises, and explore the relationship between the immersive user experience and users' intention of exercising.
\end{abstract}

Keywords: Immersive experience $\cdot$ Data visualization $\cdot$ Social interaction · Indoor exercising

\section{Introduction}

\subsection{Background}

Regular exercising in daily life, such as running, cycling and swimming, is considered to be beneficial for people in both physical and mental health.

To increase the efficiency of exercise, large numbers of professional fitness machines have been invented to help in controlling the quality of posture and movement, improving the pertinence of individuals' fitness plans, and saving our time.

However, besides the benefits brought by the machines, there are also varieties of drawbacks, especially from the perspective of user experience, such as being stuck indoor with unchanged environment, getting abstract and confusing data as feedback, and feeling being isolated by the machines.

These disadvantages could gradually drive regular exercising in recent days into a lonely, boring and repeated process. It is hard for many to carrying on doing the exercises for a longer period of time. Boredom, loneliness and no obvious progress are common excuses for many to quit their exercises for fitness.

A better context for people to keep regular exercising is to experience an enjoyable or interesting process with their friends together at the same place, which is usually difficult to accomplish in real life. It gives opportunities for research and design practice to explore new concepts and solutions to improve current situations. 


\subsection{Related Work}

A lot of studies have been done to improve the indoor exercising experience. Currently, there are two typical research directions: one is to simulate the real sports environment or create virtual immersive environment [1,2]; the other is to combine the exercises with (online) gaming elements [3]. There are also improvements on extended service like long-term data recording and analysis.

Simulation of the real environment helps to build up an immersive experience with familiar outdoor scenes in exercising, and enriches the experience by adding natural factors onto indoor training. For example, some cycling machines provide different exercising modes to simulate outdoor contexts like climbing a mountain or riding along a road. ${ }^{1}$ The creation of virtual immersive environment can provide people with more engaged and fresh experience that they may not have indoor in real life. However, these research directions seldom focus on creating social connection for users.

The direction to combine indoor exercising with game factors, especially online game modes, focuses more on facilitating social interaction and creating playful experience through gaming mechanics, ${ }^{2}$ while the exercising quality is no longer a core of the sporting process.

With the maturity of sensing technology and social media, applications and services have been implemented to motivate people to do exercises through making use of psychological factors in competitions. These products and systems also provide users new approaches to record and share their achievements with others, and are becoming increasingly intelligent in giving appropriate advices based on the analysis of long-term exercising records. ${ }^{3}$ Yet, these design concepts suit more in providing support services rather than improving the real-time experience during the exercises.

Based on observation on related work, it indicates that there is still a need to explore more possibilities for improving indoor exercising experience and evoking users' intention of exercising in places like gymnasium and sports center.

Compared with existing solutions, the design project presented here pays more attention to creating a real-time and immersive experience in digitally augmented indoor environment. It focuses on two major design problems: one is how to create an immersive but functional exercising experience, which can transform the abstract numbers from the sensors into understandable information or intuitive feelings to help users improve their exercising quality; the other is how to facilitate the sense of being accompanied through social connection and collective exercising with others.

In order to deal with the design challenges, we conducted a theoretical review to obtain a better understanding of potential methods and tools. A design framework was created and followed, aiming at bringing users a new indoor exercising experience, in order to evoke and strengthen users' intention of exercising and improve the quality of

\footnotetext{
${ }^{1}$ How to Ride Inside: Indoor Trainer Workouts for Cyclists, http://www.bicycling.com/trainingnutrition/training-fitness/how-ride-inside-indoor-trainer-workouts-cyclists.

${ }^{2}$ Play It: Soccer Becomes a Virtual Sport in Barcelona, http://www.moodmedia.se/newsdetail.asp? catid $=2 \&$ id $=3084$.

${ }^{3}$ Six running apps to keep you fit for 2012, http://www.phonesreview.co.uk/2012/05/24/6-runningapps-to-keep-you-fit-for-2012/.
} 
their exercises. A prototype was implemented based on the design framework during the hackathon event in Eindhoven, called SPRINT14. ${ }^{4}$

\section{Collective Immersive Experience}

Usually, indoor places for exercising are public, except personal environment (e.g. living room or bedroom at home), and there are lots of people in exercising, which shows potentials to provide them with a collective and immersive experience. A common method is to facilitate the immersive experience in the surrounding environment by enhancing the atmosphere around with visual or multi-modal augmentation.

\subsection{Immersive Environment for Indoor Exercises}

Immersive virtual environment (IVE) technology shows the potential in improving indoor exercising experience and enhancing the enjoyment of using. Taking advantages of virtual reality (VR), extrinsic and intrinsic motivations can be applied to evoke users' intention of exercising.

Immersion and presence are two important properties of virtual environments [4]. Since the feeling of presence is positively correlated to enjoyment [5], IVE usually emphasizes the feeling of "being there", which "requires a self-representation in the virtual environment - a virtual body" [4], according to Slater and Wilbur (1997).

While in the context of indoor exercising, users need to focus on their physical bodies, rather than the virtual body, which indicates that only digitally augmenting the environment is not enough for users who exercise regularly, as they need to pay attention to the quality of their physical exercises as well.

It is also related to users' understanding of the abstract data presented on equipment's dashboard panels. It is not so easy for people to transform the data into an intuitional feeling of how well they are doing. Even if they can understand the data after getting familiar to the functions, the reading and transforming process is still a distraction from exercising. There is a need to provide more direct or intuitive feedback to help them in real-time self-evaluation and adjustment.

Moreover, although a higher sense of presence does associate with a stronger feeling of enjoyment, it also brings possibilities in blocking social interactions and isolating users in the same indoor space.

Thus, in this context, instead of creating an immersive virtual reality environment, we tried to keep users immersed by enhancing the interplay between their exercising status and the visualization of physiological data, and to provide opportunities for social interaction. The abstract physiological data was transformed into intuitively understandable feedback through vivid and metaphorical data visualization, and projected into the physical environment, changing along with users' real-time exercising status.

\footnotetext{
${ }^{4}$ The website of SPRINT14, http://www.sprint14.nl/.
} 


\subsection{Related Study on Cognition and Mental Models}

In order to facilitate the immersive experience through visualization of the physiological data, a design framework is created based on the literature review on related notions and models, including the model of users' intention, the cognitive hierarchy, and mental model in data visualization.

Users' intention of doing exercises. Literature review on user acceptance model showed the potential of explaining the user's intention of exercising and providing a guideline for design.

Figure 1 shows the basic model that explains the relation between users' intention and their actual behaviour. It is based on the theory of planned behaviour model (TPB) [6], which is an extension of the most widely used model called theory of reasoned action (TRA) [7]. The upper blue area shows the TRA and the entire figure shows the TPB. The main difference between two models is Perceived Behavioural Control.

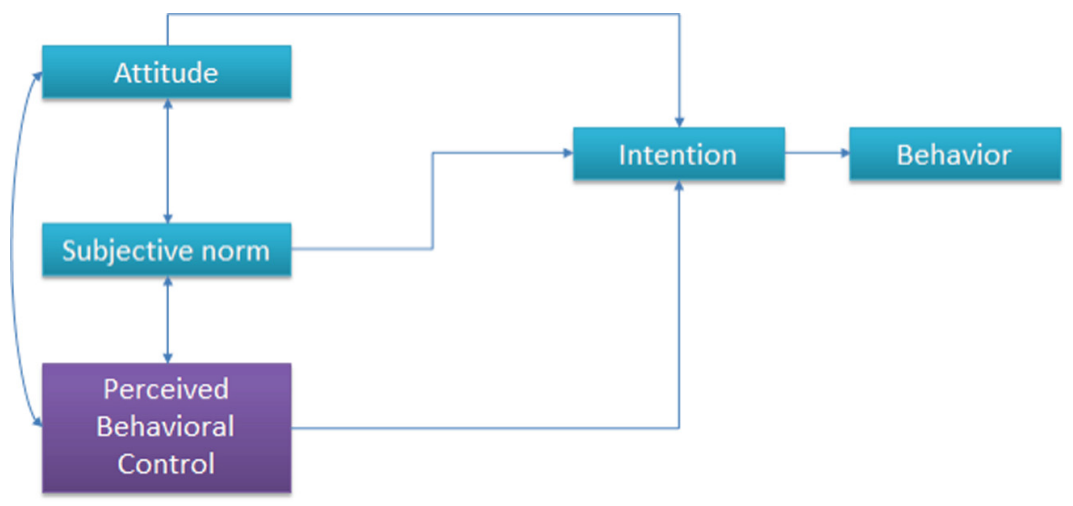

Fig. 1. Theory of reasoned action and theory of planned behaviour

Ajzen indicates that the combination of attitude towards the behaviour, subjective norms, and perception of behavioural control (Fig. 1) plays an important role in the actual behaviour execution [8]. People's subjective norms, which can be considered as "a kind of grammar of social interaction", change slowly through the social behaviour [9]. Furthermore, the easier users perceive that they can control the behaviour, the stronger their intention to perform the behaviour becomes [8].

In our context of indoor exercising, we mainly explored two design problems based on this model: (1) whether a better understanding of users' exercising status gives users a stronger perceived behaviour control, and results in stronger intention of taking the action; (2) whether the social interaction during the exercise will positively influence user's subjective norms and intention of taking the action.

Cognitive Hierarchy. Figure 2 shows the hierarchy of cognition [10], which indicates the process of knowledge generation in intelligence systems. It can be used to explain not only the process in computing system (e.g. the exercising assistance system), but also the process in human mind. 


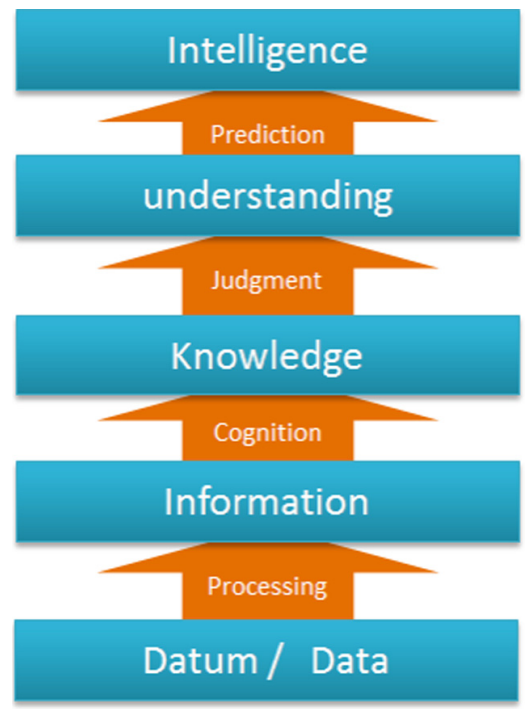

Fig. 2. The cognitive hierarchy [10]

At the bottom of Fig. 2, in the context of indoor exercising, the internal exercising data (e.g. users' physiological data) and external data (e.g. the environment data) basically present the facts, and are processed into information in the next step.

To gain knowledge from information, users' cognition works as an interpreter, translating information into knowledge, with which users can recognize the situation in a general context. When there is enough knowledge about the exercise, users can achieve an understanding of the exercising status by judging the knowledge. The final step is intelligence, which is used to support decision-making. An appropriate understanding gained from former steps will help users predict their exercising status and adjust their performance.

The cognitive hierarchy shows the process of how the external and internal data being transformed into knowledge that can help users to evaluate and adjust their performance in exercising process. And it indicates that the step of transforming information into knowledge through cognition plays an important role in obtaining good understanding to influence users' intention of exercising.

Mental models for data visualization. Considering the cognitive hierarchy mentioned above, current information presentation applied by many existing indoor exercising systems leaves a gap between information and knowledge. Most data cannot be made full use of in a smooth and continuous process. While it is suggested in many related studies that mental models can work as a joint in between [11, 12].

Mental models have been defined as an internal representation of concepts, which is considered to be critical for cognition theories. Since they are mental structures, they are used to infer novel knowledge, which is the deeper understanding of presented information [13], and have strong influences on users' knowledge construction. In visual analytics process [11], abstract knowledge is generated from information by 
users' mental models. In the case of indoor exercising system design, mental models will be engaged for ensuring that understandable knowledge is provided to users.

\subsection{Design Framework}

Based on the combination of mentioned models, we propose a design framework for indoor exercising (Fig. 3). In this framework, data has been divided into two categories, and information will be processed into different potential knowledge.

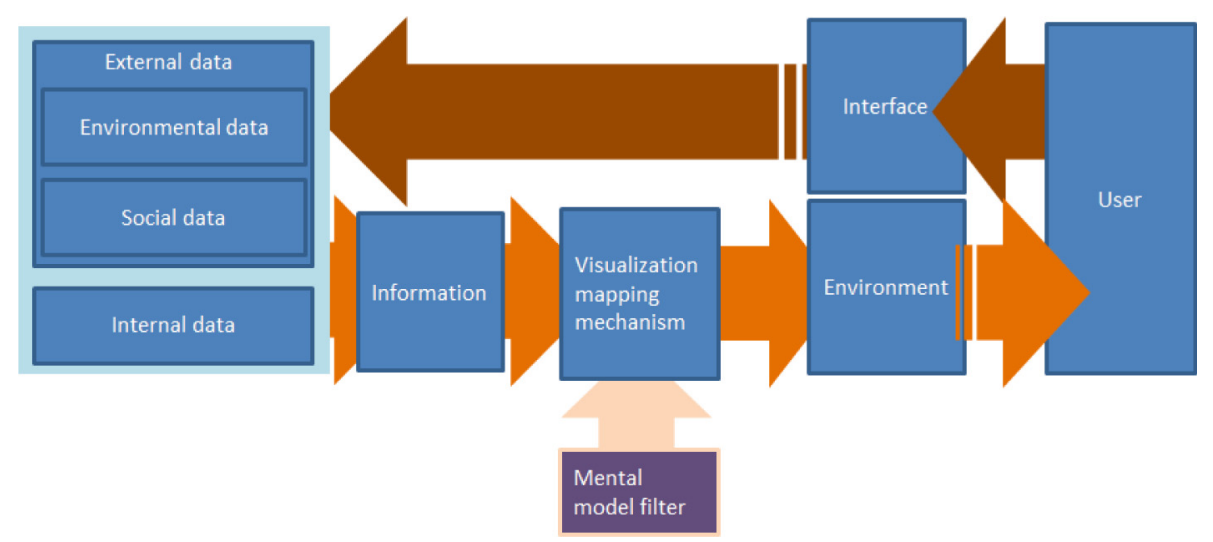

Fig. 3. Design framework for indoor exercises

Internal data will be collected to present the exercising status, such as users' heart rate and the calories burned during the exercises. External data, including both the indoor environmental data and users' social data, will also be collected to help project visualized information into the physical space and create potential social connection between users.

Users' mental models are used to create a mental model filter, and a visualization mapping mechanism is designed for more appropriate data transformation and knowledge generation based on this filter. The generated knowledge will be projected into the physical environment to create an immersive experience. Since the knowledge is generated by the mapping mechanism, based on users' mental models, the understanding should be more suitable and accurate. After obtaining a better understanding of the exercising status, users can better adjust their performance and interact with others though the visualization in the environment.

\section{Project RIPPLE}

\subsection{Concept Implementation}

Concept for prototype. The concept "RIPPLE" entails an interactive immersive environment for cycling exercises. The goal of the project is to offer a better user 
experience by creating an immersive environment and an interactive visualization. The prototype was initially constructed during a two-day hackathon event, called SPRINT14 in Eindhoven, and became one of the winning concepts to be further developed, due to the novel immersive experience and the delighting social connection it might bring to users.

The metaphorical data visualization is employed to present users' exercising status and exercise-related physiological data (e.g. heart rate and calories). Compared to the traditional graphic or numeric display, the interactive visualization is assumed to feedback the sport and bodily information to the users in a more intuitional and understandable way. In multi-user contexts, a social connection is built up between users in the same space through the dynamic changes of the elements in the visualization.

Visualization Design. As shown in Fig. 4, we selected the 'sea' as the background of the visualization; it renders a nature scene in an indoor space. The size and amount of waves under the cycling machine are mapped from the cycling speed, which creates a dramatic effect, as if the user is riding across the surface of the water. The ripples are triggered by the user's heartbeats. The idea here is to present a sweat-breaking process of cycling exercises. As the intensity and the duration of the exercise increasing, increased heart rate will generate more ripples in the water.
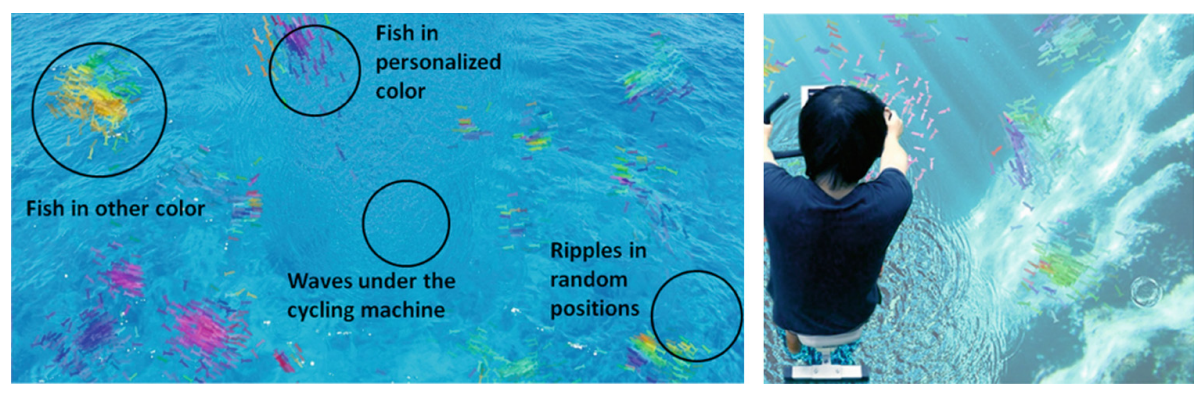

Fig. 4. Visualization design in the project

To promote social bonding or connections in a 'light-weight' way with little extra operation and distraction from exercising, we use the "abstract fish" as the symbol of users; the amount of the fish of one user will be mapped to the calories she burnt in the exercise. The fish appear around the user initially, and as the exercising time passes by, the fish will swim outside to 'communicate' with the fish of other users. This process is designed to create a social bonding and a collective exercising experience, breaking the individuals' isolated status and providing users with more fun and motivation.

Data collection. In this prototype, a phytoplethysmograph (PPG) sensor is used to measure pulse signals from the participant's index finger. Compared to ECG electrode, the biggest advantage of the optimal PPG is being easy to use. Participants do not need to attach gel-coated electrodes on their chest. Heart rate data is calculated from pulse signal and used in in real-time. The calories burned during the exercise session are 
estimated by using the following formula: $\mathrm{C}=(0.4472 \times \mathrm{H}-0.05741 \times \mathrm{W}+0.074 \times$ $\mathrm{A}-20.4022) \times \mathrm{T} / 4.184$ [14]. $\mathrm{C}$ is the number of calories burned, $\mathrm{H}$ is the average heart rate, $\mathrm{W}$ is the weight, $\mathrm{A}$ is the user' age and $\mathrm{T}$ is the length of the exercise session in minutes.

Set-up. In order not to interrupt the exercise process, the visualization is projected on the floor, under the cycling machine (Fig. 5). A projector is fixed on a frame above and in front of the cycling machine. A cycling machine is placed on a white curtain tiled on the ground. The visualization program is developed in Processing.
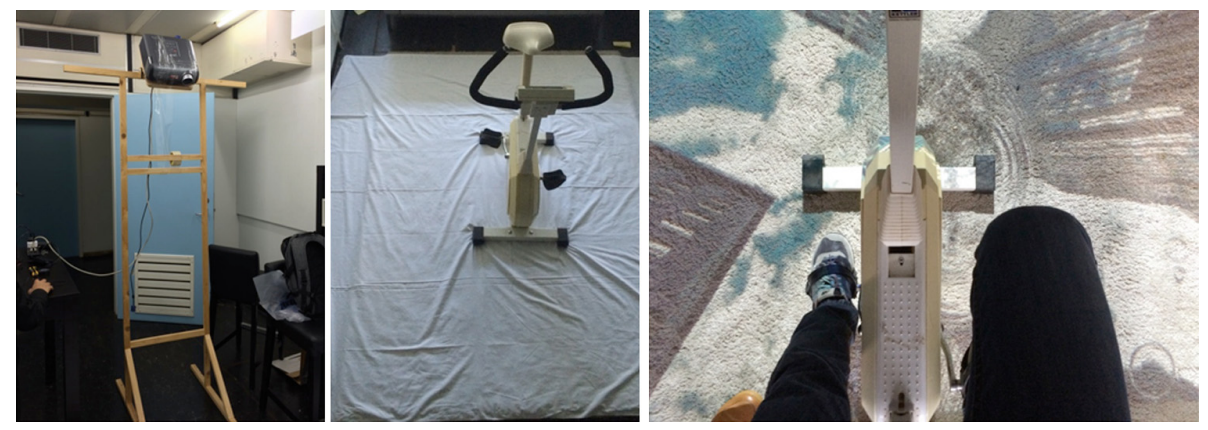

Fig. 5. The set-up of the 'Ripple'

\subsection{Social Interaction Through Exercising Data}

Besides the real-time interplay between users and the visualized data, the potential social connection between users is also explored, in order to create a collective exercising experience, breaking the individuals' isolated status and providing users with more fun and motivation.

Since the context of doing regular exercises with friends together is hard to realize for many in daily life, design concepts are explored, for example, facilitating interaction between people who are doing exercise at the same place.

The indoor exercising space holds the potential to provide users with a social environment, while it is usually hard for strangers to break the ice and socially interact with each other. There is a need to help them create the connection that will neither make them feel embarrassed nor interrupt their exercises.

Compared with the solutions that connect friends online for gaming or communication, we prefer to enhance the connection at same indoor places, based on the physiological data visualization.

The social interaction in this context is to connect users by facilitating cooperation through the visualization, raising social awareness between people via its lively movements and changes [15], and providing a collective feeling of being accompanied or appreciated in exercising. When people are connected, the interaction between their data and the impact brought towards the whole augmented environment will change along with the cooperation. For example, if all the users are exercising in a standard or high-quality status, the collective visualization may transform into a more harmonic presentation as a reward or general feedback for their efforts. 


\subsection{Next Steps}

The design concept introduced in this paper tries to transform the indoor space into an immersive environment for exercising people through ambient projection. The whole design aims to help users concentrate on their exercising while being aware of their exercising status (e.g. standard or not).

In addition, instead of connecting with friends online to socially interact through avatars or images, we explore the possibilities to highlight or create social connection between the people who are doing co-located exercising. The social interaction between users and the immersive environment provide users intensive enjoyment, which can be considered as an intrinsic motivation for taking regular exercises.

For the next steps, iterations of the design framework and the prototype will be conducted, in order to deploy further design research on the relation between the immersive experience and users' engagement and motivation towards indoor exercising.

The mechanism of mental models needs to be further developed and tested, in order to work as an instrument for knowledge selection and visualization mapping in a certain context.

The long-term data recording may be added into the system for a better self-evaluation and real-time analysis of users' exercising status. The recording can also be used for socially motivating users by combining it with current social service.

\section{Conclusion}

In the presented project, we focus on creating an immersive experience through data visualization, creating and enhancing social interactions between co-located exercising people.

To evoke and strengthen users' intention of exercising, literature study on cognitive psychology, immersive virtual reality, and user acceptance was conducted. A design framework was used to guide the visualization design process for indoor exercising context, such as gymnasium and sport center. A prototype was implemented based on the design framework, using the sea and fish as metaphor to create an interactive immersive environment for indoor cycling context.

Through this design, we hope to create an interesting, motivating and unique experience for indoor exercising through data visualization and ambient projection in physical space.

Acknowledgments. We would like to thank China Scholarship Council for the support on the design research. We also thank MADspace Eindhoven and InnoSportLab Sport en Beweeg and all the sponsors of SPRINT14 for the support on the event.

\section{References}

1. Poussard, B., et al.: 3DLive: a multi-modal sensing platform allowing tele-immersive sports applications. In: 22nd Signal Processing Conference (EUSIPCO), pp. 356-360. IEEE: Lisbon, Portugal (2014) 
2. Pallot, M., et al.: Augmented sport: exploring collective user experience. In: Virtual Reality International Conference: Laval Virtual, pp. 1-8. ACM: Laval, France (2013)

3. Mueller, F., Agamanolis, S., Picard, R.: Exertion interfaces: sports over a distance for social bonding and fun. In: SIGCHI Conference on Human Factors in Computing Systems, pp. 561-568. ACM: Ft. Lauderdale, Florida, USA (2003)

4. Slater, M., Wilbur, S.: A framework for immersive virtual environments (FIVE): speculations on the role of presence in virtual environments. Teleoperators Virtual Environ. 6, 603-616 (1997)

5. Ijsselsteijn, W.A., de Kort, Y.A.W., Westerink, J., de Jage, M.: Virtual fitness: stimulating exercise behavior through media technology. Presence Teleoperators Virtual Environ. 15(6), 688-698 (2006). MIT Press

6. Ajzen, I.: The theory of planned behavior. Organ. Behav. Hum. Decis. Process. 50(2), 179211 (1991)

7. Fishbein, M., Ajzen, I.: Belief, Attitude, Intention and Behavior: An Introduction to Theory and Research. Addison-Wesley, Reading (1975)

8. Ajzen, I.: Constructing a theory of planned behavior questionnaire (2014). http://www. researchgate.net/publication/235913732_Constructing_a_Theory_of_Planned_Behavior_ Questionnaire

9. Bicchieri, C., Muldoon, R.: Social Norms, Stanford Encyclopedia of Philosophy. http:// plato.stanford.edu/entries/social-norms/\#Con

10. Cox, J.: Intelligence: Definitions, Concepts and Governance. Parliamentary Information and Research Service, Library of Parliament (2009)

11. Liu, Z., Stasko, J.T.: Mental models, visual reasoning and interaction in information visualization: a top-down perspective. Vis. Comput. Graph. 16(6), 999-1008 (2010). IEEE

12. Mueller, K., Garg, S., et al.: Can computers master the art of communication? a focus on visual analytics. IEEE Comput. Graph. Appl. 3(31), 14-21 (2011). IEEE

13. Rapp, D.N.: Mental Models: Theoretical Issues for Visualizations in Science Education. Models and Modeling in Science Education, pp. 43-60. Springer, Netherlands (2005)

14. Keytel, L.R., et al.: Prediction of energy expenditure from heart rate monitoring during submaximal exercise. J. Sports Sci. 23(3), 289-297 (2005)

15. Hu, J., Le, D., Funk, M., Wang, F., Rauterberg, M.: Attractiveness of an interactive public art installation. In: Streitz, N., Stephanidis, C. (eds.) DAPI 2013. LNCS, vol. 8028, pp. 430438. Springer, Heidelberg (2013) 


\section{P.L. Patrick Rau (Ed.)}

\section{Cross-Cultural Design}

\section{Applications in Mobile Interaction,}

Education, Health, Transport and Cultural Heritage

7th International Conference, CCD 2015 Held as Part of $\mathrm{HCl}$ International 2015 Los Angeles, CA, USA, August 2-7, 2015, Proceedings, Part II
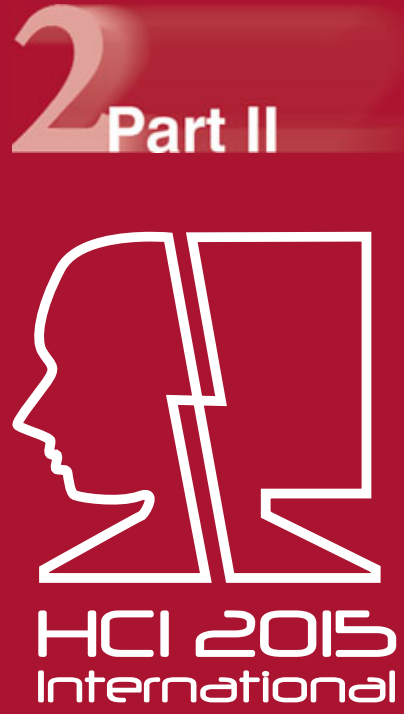

Springer 


\section{Lecture Notes in Computer Science}

Commenced Publication in 1973

Founding and Former Series Editors:

Gerhard Goos, Juris Hartmanis, and Jan van Leeuwen

\section{Editorial Board}

David Hutchison

Lancaster University, Lancaster, UK

Takeo Kanade

Carnegie Mellon University, Pittsburgh, PA, USA

Josef Kittler

University of Surrey, Guildford, UK

Jon M. Kleinberg

Cornell University, Ithaca, NY, USA

Friedemann Mattern

ETH Zurich, Zürich, Switzerland

John C. Mitchell

Stanford University, Stanford, CA, USA

Moni Naor

Weizmann Institute of Science, Rehovot, Israel

C. Pandu Rangan

Indian Institute of Technology, Madras, India

Bernhard Steffen

TU Dortmund University, Dortmund, Germany

Demetri Terzopoulos

University of California, Los Angeles, CA, USA

Doug Tygar

University of California, Berkeley, CA, USA

Gerhard Weikum

Max Planck Institute for Informatics, Saarbrücken, Germany 
More information about this series at http://www.springer.com/series/7409 
P.L. Patrick Rau (Ed.)

\section{Cross-Cultural Design}

Applications in Mobile Interaction, Education, Health, Transport and Cultural Heritage

7th International Conference, CCD 2015

Held as Part of HCI International 2015

Los Angeles, CA, USA, August 2-7, 2015

Proceedings, Part II

黛 Springer 
Editor

P.L. Patrick Rau

Department of Industrial Engineering

Tsinghua University

Beijing

P.R. China

ISSN 0302-9743

ISSN 1611-3349 (electronic)

Lecture Notes in Computer Science

ISBN 978-3-319-20933-3

ISBN 978-3-319-20934-0 (eBook)

DOI 10.1007/978-3-319-20934-0

Library of Congress Control Number: 2015943044

LNCS Sublibrary: SL3 - Information Systems and Applications, incl. Internet/Web, and HCI

Springer Cham Heidelberg New York Dordrecht London

(C) Springer International Publishing Switzerland 2015

This work is subject to copyright. All rights are reserved by the Publisher, whether the whole or part of the material is concerned, specifically the rights of translation, reprinting, reuse of illustrations, recitation, broadcasting, reproduction on microfilms or in any other physical way, and transmission or information storage and retrieval, electronic adaptation, computer software, or by similar or dissimilar methodology now known or hereafter developed.

The use of general descriptive names, registered names, trademarks, service marks, etc. in this publication does not imply, even in the absence of a specific statement, that such names are exempt from the relevant protective laws and regulations and therefore free for general use.

The publisher, the authors and the editors are safe to assume that the advice and information in this book are believed to be true and accurate at the date of publication. Neither the publisher nor the authors or the editors give a warranty, express or implied, with respect to the material contained herein or for any errors or omissions that may have been made.

Printed on acid-free paper

Springer International Publishing AG Switzerland is part of Springer Science+Business Media (www.springer.com) 


\section{Foreword}

The 17th International Conference on Human-Computer Interaction, HCI International 2015, was held in Los Angeles, CA, USA, during 2-7 August 2015. The event incorporated the 15 conferences/thematic areas listed on the following page.

A total of 4843 individuals from academia, research institutes, industry, and governmental agencies from 73 countries submitted contributions, and 1462 papers and 246 posters have been included in the proceedings. These papers address the latest research and development efforts and highlight the human aspects of design and use of computing systems. The papers thoroughly cover the entire field of Human-Computer Interaction, addressing major advances in knowledge and effective use of computers in a variety of application areas. The volumes constituting the full 28-volume set of the conference proceedings are listed on pages VII and VIII.

I would like to thank the Program Board Chairs and the members of the Program Boards of all thematic areas and affiliated conferences for their contribution to the highest scientific quality and the overall success of the HCI International 2015 conference.

This conference could not have been possible without the continuous and unwavering support and advice of the founder, Conference General Chair Emeritus and Conference Scientific Advisor, Prof. Gavriel Salvendy. For their outstanding efforts, I would like to express my appreciation to the Communications Chair and Editor of HCI International News, Dr. Abbas Moallem, and the Student Volunteer Chair, Prof. Kim-Phuong L. Vu. Finally, for their dedicated contribution towards the smooth organization of HCI International 2015, I would like to express my gratitude to Maria Pitsoulaki and George Paparoulis, General Chair Assistants. 


\section{HCI International 2015 Thematic Areas and Affiliated Conferences}

Thematic areas:

- Human-Computer Interaction (HCI 2015)

- Human Interface and the Management of Information (HIMI 2015)

Affiliated conferences:

- 12th International Conference on Engineering Psychology and Cognitive Ergonomics (EPCE 2015)

- 9th International Conference on Universal Access in Human-Computer Interaction (UAHCI 2015)

- 7th International Conference on Virtual, Augmented and Mixed Reality (VAMR 2015)

- 7th International Conference on Cross-Cultural Design (CCD 2015)

- 7th International Conference on Social Computing and Social Media (SCSM 2015)

- 9th International Conference on Augmented Cognition (AC 2015)

- 6th International Conference on Digital Human Modeling and Applications in Health, Safety, Ergonomics and Risk Management (DHM 2015)

- 4th International Conference on Design, User Experience and Usability (DUXU 2015)

- 3rd International Conference on Distributed, Ambient and Pervasive Interactions (DAPI 2015)

- 3rd International Conference on Human Aspects of Information Security, Privacy and Trust (HAS 2015)

- 2nd International Conference on HCI in Business (HCIB 2015)

- 2nd International Conference on Learning and Collaboration Technologies (LCT 2015)

- 1st International Conference on Human Aspects of IT for the Aged Population (ITAP 2015) 


\section{Conference Proceedings Volumes Full List}

1. LNCS 9169, Human-Computer Interaction: Design and Evaluation (Part I), edited by Masaaki Kurosu

2. LNCS 9170, Human-Computer Interaction: Interaction Technologies (Part II), edited by Masaaki Kurosu

3. LNCS 9171, Human-Computer Interaction: Users and Contexts (Part III), edited by Masaaki Kurosu

4. LNCS 9172, Human Interface and the Management of Information: Information and Knowledge Design (Part I), edited by Sakae Yamamoto

5. LNCS 9173, Human Interface and the Management of Information: Information and Knowledge in Context (Part II), edited by Sakae Yamamoto

6. LNAI 9174, Engineering Psychology and Cognitive Ergonomics, edited by Don Harris

7. LNCS 9175, Universal Access in Human-Computer Interaction: Access to Today's Technologies (Part I), edited by Margherita Antona and Constantine Stephanidis

8. LNCS 9176, Universal Access in Human-Computer Interaction: Access to Interaction (Part II), edited by Margherita Antona and Constantine Stephanidis

9. LNCS 9177, Universal Access in Human-Computer Interaction: Access to Learning, Health and Well-Being (Part III), edited by Margherita Antona and Constantine Stephanidis

10. LNCS 9178, Universal Access in Human-Computer Interaction: Access to the Human Environment and Culture (Part IV), edited by Margherita Antona and Constantine Stephanidis

11. LNCS 9179, Virtual, Augmented and Mixed Reality, edited by Randall Shumaker and Stephanie Lackey

12. LNCS 9180, Cross-Cultural Design: Methods, Practice and Impact (Part I), edited by P.L. Patrick Rau

13. LNCS 9181, Cross-Cultural Design: Applications in Mobile Interaction, Education, Health, Transport and Cultural Heritage (Part II), edited by P.L. Patrick Rau

14. LNCS 9182, Social Computing and Social Media, edited by Gabriele Meiselwitz

15. LNAI 9183, Foundations of Augmented Cognition, edited by Dylan D. Schmorrow and Cali M. Fidopiastis

16. LNCS 9184, Digital Human Modeling and Applications in Health, Safety, Ergonomics and Risk Management: Human Modeling (Part I), edited by Vincent G. Duffy

17. LNCS 9185, Digital Human Modeling and Applications in Health, Safety, Ergonomics and Risk Management: Ergonomics and Health (Part II), edited by Vincent G. Duffy

18. LNCS 9186, Design, User Experience, and Usability: Design Discourse (Part I), edited by Aaron Marcus

19. LNCS 9187, Design, User Experience, and Usability: Users and Interactions (Part II), edited by Aaron Marcus

20. LNCS 9188, Design, User Experience, and Usability: Interactive Experience Design (Part III), edited by Aaron Marcus 
21. LNCS 9189, Distributed, Ambient and Pervasive Interactions, edited by Norbert Streitz and Panos Markopoulos

22. LNCS 9190, Human Aspects of Information Security, Privacy and Trust, edited by Theo Tryfonas and Ioannis Askoxylakis

23. LNCS 9191, HCI in Business, edited by Fiona Fui-Hoon Nah and Chuan-Hoo Tan

24. LNCS 9192, Learning and Collaboration Technologies, edited by Panayiotis Zaphiris and Andri Ioannou

25. LNCS 9193, Human Aspects of IT for the Aged Population: Design for Aging (Part I), edited by Jia Zhou and Gavriel Salvendy

26. LNCS 9194, Human Aspects of IT for the Aged Population: Design for Everyday Life (Part II), edited by Jia Zhou and Gavriel Salvendy

27. CCIS 528, HCI International 2015 Posters' Extended Abstracts (Part I), edited by Constantine Stephanidis

28. CCIS 529, HCI International 2015 Posters' Extended Abstracts (Part II), edited by Constantine Stephanidis 


\section{Cross-Cultural Design}

\section{Program Board Chair: P.L. Patrick Rau, P.R. China}

- Zhe Chen, P.R. China

- Yu-Liang Chi, Taiwan

- Paul L. Fu, USA

- Zhiyong Fu, P.R. China

- Sung H. Han, Korea

- Toshikazu Kato, Japan

- Pin-Chao Liao, P.R. China

- Dyi-Yih Michael Lin, Taiwan

- Rungtai Lin, Taiwan
- Jun Liu, P.R. China

- Yongqi Lou, P.R. China

- Ta-Ping Lu, Taiwan

- Liang Ma, P.R. China

- Alexander Mädche, Germany

- Sheau-Farn Max Liang, Taiwan

- Katsuhiko Ogawa, Japan

- Huatong Sun, USA

- Hsiu-Ping Yueh, Taiwan

The full list with the Program Board Chairs and the members of the Program Boards of all thematic areas and affiliated conferences is available online at:

http://www.hci.international/2015/

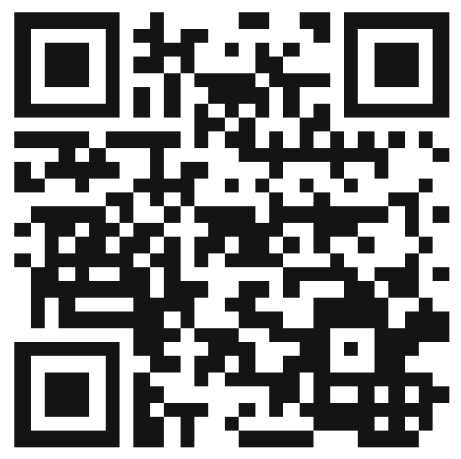




\section{HCI International 2016}

The 18th International Conference on Human-Computer Interaction, HCI International 2016, will be held jointly with the affiliated conferences in Toronto, Canada, at the Westin Harbour Castle Hotel, 17-22 July 2016. It will cover a broad spectrum of themes related to Human-Computer Interaction, including theoretical issues, methods, tools, processes, and case studies in HCI design, as well as novel interaction techniques, interfaces, and applications. The proceedings will be published by Springer. More information will be available on the conference website: http://2016.hci.international/.

\section{General Chair}

Prof. Constantine Stephanidis

University of Crete and ICS-FORTH

Heraklion, Crete, Greece

Email: general_chair@hcii2016.org

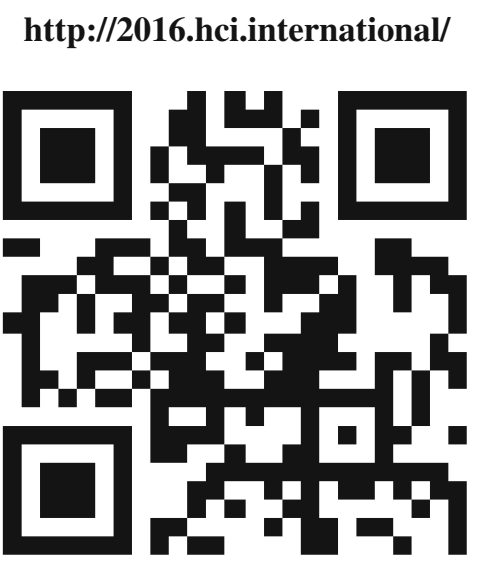




\section{Contents - Part II}

\section{Cultural Aspects of Social Media and Mobile Services}

Culturally Appropriate Design of Mobile Learning Applications

in the Malaysian Context . . . . . . . . . . . . . . . . . . . . . . . . . . . . . .

Shamsul Arrieya Ariffin and Laurel Evelyn Dyson

How Online Social Network and Wearable Devices Enhance Exercise

Well-Being of Chinese Females? . . . . . . . . . . . . . . . . . .

Hao Chen, Ting-Yu Tony Lin, Qiaochu Mu, and Pei-Luen Patrick Rau

Social Media Design Requirements for the Collectivist

International Students

Kanrawi Kitkhachonkunlaphat and Mihaela Vorvoreanu

"Faith to Go or Devil's Work" - Social Media Acceptance

in Taboo-Related Usage Contexts . . . . . . . . . . . . . . . . . . . . .

Judith Leckebusch, Sylvia Kowalewski, Chantal Lidynia, and Martina Ziefle

The Impact of Natural Utilization of Traditional Chinese Cultural Elements on the User Experience in Mobile Interaction Design . . . . . . . . . . . . .

Tian Lei, Xu Liu, Lei Wu, Tianjian Chen, Yuhui Wang, Luyao Xiong, and Shuaili Wei

Service Design Towards Sustainable Lifestyle in the Context of Mobile Internet . . . . . . . . . . . . . . . . . . . . . .

Xueliang Li, Miaosen Gong, and Dongjuan Xiao

From Technology to Design: A Case Study of Netizen's Perception Toward Dechnology Products. . . . . . . . . . . . . . . . . . . . .

Hsi-Yen Lin, Wen-Zhong Su, Pei-Hua Hung, and Chi-Ying Hung

From Customer Satisfaction to Customer Experience: Online Customer Satisfaction Practice in International E-commerce . . . . . . . . . . . . . . . Yanyang Liuqu, Xinheng Fan, and Paul L. Fu

E-Commerce Purchase Intention in Emerging Markets: The Influence

of Gender and Culture . . . . . . . . . . . . . . . . . . . . . . .

Dimitrios Rigas and Nazish Riaz.

Cultural Capital at Work in Facebook Users' Selection of Different Languages . . . . . . . . . . . . . . . . . . . . . . . Jieyu Wang and Satarupa Joardar 


\section{Culture for Transport and Travel}

Applying Soundscape to Creating an Interactive and Cultural

Centered Experience . . . . . . . . . . . . . . . . . . . .

Hsiu Ching Laura Hsieh and Chiao Yu Hwang

Design of Vehicle-to-Vehicle Communication System for Chinese and German Drivers . . . . . . . . . . . . . . . . . . . . .

Xiang Ji, Lukas Haferkamp, Chieh Cheng,

Muanphet Charunratanavisan, Andreas Neuhaus, Na Sun, and Pei-Luen Patrick Rau

Investigation of a Driver-to-Driver Communication Method

Through Rear Window Display for Chinese . . . . . . . . . . . . . . .

Na Liu, Ruifeng Yu, Deyu Wang, and Yunhong Zhang

On the Qualitative Research Approach and Application

of the "VTIO" Model Based on Cultural Differences:

A Case Study of Changan Ford Mazda Automobile Co., Ltd . . . . . . . . . . .

Lei Liu and Lin Ma

Driving Safety Considered User Interface of a Smartphone:

An Experimental Comparison . . . . . . . . . . . . . . . . . .

Sanaz Motamedi, Mahdi Hasheminejad, and Pilsung Choe

Exploring Smart-Car Space in Urban India. . . . . . . . . . . . . . . .

Sarita Seshagiri and Aditya Ponnada

Ask Local: Explore a New Place Like Locals . . . . . . . . . . . . . . . . . . .

Cagri Hakan Zaman, Federico Casalegno, Meng Sun, and Kulpreet Chilana

\section{Culture for Design and Design for Culture}

Analysis of Emotional Design and Cultural Product Narrative

Communication Model. . . . . . . . . . . . . . . . . . . . . . . . .

Miao-Hsien Chuang and Jui-Ping Ma

From Design to Humanity - A Case Study of Costumer Value

Toward Dechnology Products. . . . . . . . . . . . . . . . .

Chi-Ying Hung, Pei-Hua Hung, Wen-Zhong Su, and Hsi-Yen Lin

From Design to Technology: A Case Study of Children's Perception

Toward the Dechnology Products . . . . . . . . . . . . . . . .

Pei-Hua Hung, Chi-Ying Hung, Hsi-Yen Lin, and Wen-Zhong Su

Monster Design and Classifier Cognition . . . . . . . . . . . . . . . Larry Hong-lin Li 
Design of Literature Management Tool . . . . . . . . . . . . . . . Xiaojing Liao

Emotion and Perception: A Case Study of Aesthetic Response

to Frith's Narrative Painting "The Railway Station".

Po-Hsien Lin, Mo-Li Yeh, and Jao-Hsun Tseng

Traditional Western Art Elements in Disney Animations, Elite Influence

in Mass Culture Through the Prism of the Frankfurt School . . . . . . . . . . Nai-Hsuan Lin and Shwu-Huoy Tzou

From Dechnology to Humart - A Case Study of Taiwan Design

Development . . . . . . . . . . . . . . . . . . . .

Rungtai Lin, John Kreifeldt, Pei-Hua Hung, and Jun-Liang Chen

Human Factors Perspective of Dancing Props Design: A Case Study

of "Feiyan's Dancing on Palms".....................

Jao-Hsun Tseng and Po-Hsien Lin

The Application of Chinese Poem "Yu Mei Ren" in Design . . . . . . . . . . .

Mo-Li Yeh, Hsi-Yen Lin, Ming-shean Wang, and Rungtai Lin

Cultural Identification and Innovation-A Study on the Design of Exhibition and Dissemination System for a City's Cultural Heritage

Under the New Media Context . . . . . . . . . . . . . . . . . . .

Lie Zhang and Wen Zhang

\section{Culture for Health, Learning and Games}

Paper Catalog and Digital Catalog - Reading Behaviors of College Students

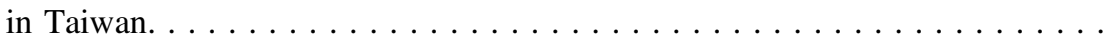

Yu-Ju Lin, Hui-Yun Yen, Chiui Hsu, Yige Jin, and Po-Hsien Lin

"Break the Language Great Wall" (RedClay): The Language Learning Application . . . . . . . . . . . . . . . . . . . .

Ting-Yu Tony Lin, Benoit Serot, Maxime Verlhac, Marie Maniglier, Na Sun, and Pei-Luen Patrick Rau

Interact Through Your Data: Collective Immersive Experience Design

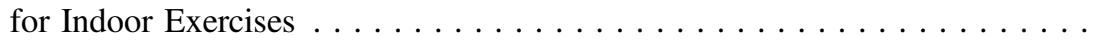

Xu Lin, Linkai Tao, Bin Yu, Yongyan Guo, and Jun Hu

Leap-Motion Based Online Interactive System for Hand Rehabilitation . . . . . Zhe Liu, Yingzhi Zhang, Pei-Luen Patrick Rau, Pilsung Choe, and Tauseef Gulrez. 
From Dechnology to Humart: A Case Study of Applying Nature

User Interface to the Interactive Rehabilitation Design . . . . . . . . . . . . . . 348 Jui Ping Ma, Na Ling Huang, Miao Hsien Chuang, and Rungtai Lin

Physician Communication Behaviors that Predict Patient Trust in Outpatient Departments . . . . . . . . . . . . . . . . . . 361

Manrong She, Zhizhong Li, and Pei-Luen Patrick Rau

Cultural Difference on Team Performance Between Chinese and Americans in Multiplayer Online Battle Arena Games. . . . . . . . . . . . . . . . . . . . . . 374 Huiwen Wang, Bang Xia, and Zhe Chen

Defining Design Opportunities of Healthcare in the Perspective of Digital Social Innovation . . . . . . . . . . . . . . . . . . . . . . 384 Dongjuan Xiao, Miaosen Gong, and Xueliang $\mathrm{Li}$

The Service System Study on Children's Hospital-Bed Nursing Based on Multi-level Experience. . . . . . . . . . . . . . . . . . . . . . . Linghao Zhang, Chang Zhang, Sheng Huang, and Sichun Xiao

Field Study on College Students' Uses and Gratifications of Multitasking Interaction with Multiple Smart Devices. . . . . . . . . . . . . Yubo Zhang and Pei-Luen Patrick Rau 


\section{Contents - Part I}

\section{Cross-Cultural Product Design}

A Brand Construction Strategy of Digital Cultural and Creative Empowerment in Local Cultural Industries . . . . . . . . . . . . . . .

Tsen-Yao Chang and Kuo-Li Huang

The Effects of Form Ratio in Product Design . . . . . . . . . . . . . . .

Chiu-Wei Chien, Chih-Long Lin, and Rungtai Lin

Pilot Study on the Application of Light-Absorbing Fabric in Sport Fashion. .

Chiui Hsu and Po-Hsien Lin

A Design Strategy of Cultural and Creative Products on the Global Market. . Chi-Hsien Hsu and Wang-Chin Tsai

Chinese Cultural Values in User Experience Design of Kids' Home

Products. . . . . . . . . . . . . . . . . . . . . . . . . .

Xiaojun Huang and Linong Dai

Cross-Cultural User Experience Design Helping Product Designers

to Consider Cultural Differences . . . . . . . . . . . . . . . . . . . . . .

Florian Lachner, Constantin von Saucken, Florian 'Floyd' Mueller, and Udo Lindemann

A Product Service System Design for Fitness Activities Based on Active Ageing a Proposal of Fitness for Xishan Style Council . . . . . . . . . . . .

Yin Liang and Davide Fassi

A Study of the Accessible Approach to Replace the Reservoir Silt Glaze with New Formula

Chi-Chang Lu and Po-Hsien Lin

Humanism Presented in Taiwan Cochin Ceramic Design . . . . . . . . . . . Huei-Mei Shih

Designing Wearable Device-Based Product and Service Ecosystem . . . . . . . . 108 Xiaohua Sun, Yongqi Lou, Tong Li, and Qi Wang

From OEM to OBM - A Case Study of Branding Taiwan . . . . . . . . . . .

Hui-Yun Yen, Yu-Ju Lin, Yige Jin, and Rungtai Lin 
Research on Service-Driven Feature of Industrial Designers Under the Background of Industry Convergence. . . . . . . . . . . . . .

Qing Zhang, Chen Cheng, Junnan Ye, and Wei Ding

\section{Cross-Cultural Design Methods and Case Studies}

Comparison of User Responses to English and Arabic Emotion Elicitation Video Clips . . . . . . . . . . . . . . . . . . . . . . .

Nawal Al-Mutairi, Sharifa Alghowinem, and Areej Al-Wabil

Understanding Gratifications of Watching Danmaku Videos - Videos with Overlaid Comments . . . . . . . . . . . . . . . . . . .

Yue Chen, Qin Gao, and Pei-Luen Patrick Rau

User's Individual Needs Oriented Parametric Design Method of Chinese

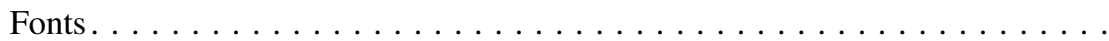

Qijun Duan and Xiaoli Zhang

Dramatic Sketches: A New Interaction Design Resource for Communicating Contextual Factors . . . . . . . . . . . . .

Fuad Ali EL-Qirem and Gilbert Cockton

A Formal Method for Evaluating the Performance Level of Human-Human Collaborative Procedures . . . . . . . . . . . . . . . . . . .

Dan Pan and Matthew L. Bolton

The Effect of Tactile Feedback on Mental Workload During the Interaction with a Smartphone . . . . . . . . . . . . . . . . . . . . .

Peter Rasche, Alexander Mertens, Christopher Schlick, and Pilsung Choe

Brazilian Cultural Differences and Their Effects on the Web Interfaces User Experience . . . . . . . . . . . . . . . . . . . . . . . .

Tales Rebequi Costa Borges de Souza, Marcelo Morandini, and João Luiz Bernardes Jr.

A Pilot Study of Exploring the Relationship Between Dechnology Product and Product Personality . . . . . . . . . . . . . . . . . . .

Wen-Zhong Su, Hsi-Yen Lin, Chi-Ying Hung, and Pei-Hua Hung

An Innovation Design for Hazardous Chemical/Gases Disaster Detection and Analysis Equipment by Using Cross-Cultural User Scenarios and Service Design . . . . . . . . . . . . . . . . . . .

Sheng-Ming Wang, Cheih Ju Huang, Lun-Chang Chou, and Pei-Lin Chen 
Based on Action-Personality Data Mining, Research of Gamification

Emission Reduction Mechanism and Intelligent Personalized Action

Recommendation Model . . . . . . . . . . . . . . . . . . . .

Yangbo $\mathrm{Xu}$ and Yi Tang

Design of a Clothing Shopping Guide Website for Visually Impaired

People...............................

Huiqiao Yang, Qijia Peng, Qin Gao, and Pei-Luen Patrick Rau

Co-design: An Investigation Through Interviewing Expert in Europe...... . Shu Yuan, Hua Dong, and Zi Chen

Investigation into Designing of Elderly Products Intending for the User's Behavior Experiencing. . . . . . . . . . . . . . . . .

Ning Zhang, Yajun Li, Ming Zhou, and Zhizheng Zhang

Research on Product Affective Image by the Way of Empathic Design . . . . . 283

Meiyu Zhou, Xiaowen Yang, Peilong Liang, and Pei Xu

\section{Design, Innovation, Social Development and Sustainability}

A Study on the Balance and Optimization Measures in Industry-University

Collaborative Innovation of Interaction Design . . . . . . . . . . . . .

Jianxin Cheng, Miao Liu, and Junnan Ye

The Research and Practice Framework for Designing the Digital Social

Innovation . . . . . . . . . . . . . . . . . . . . .

Zhiyong Fu and Zirui Huang

Defining the Middle Ground: A Comprehensive Approach to the Planning,

Design and Implementation of Smart City Operating Systems. . . . . . . . . . Christopher Grant Kirwan

Review on Interaction Design for Social Context in Public Spaces . . . . . . . Xu Lin, Jun Hu, and Matthias Rauterberg

Diagnosis on Corporate Culture and Construction: A Case Study of Limin

Chemical Co., Ltd. . . . . . . . . . . . . . . . . . . . . . . .

Lin Ma, Xueli Wang, and Xiaopeng He

When Human-Centered Design Meets Social Innovation: The Idea of Meaning Making Revisited.

Jin Ma

Design Process as Communication Agency for Value Co-Creation in Open Social Innovation Project: A Case Study of QuYang Community

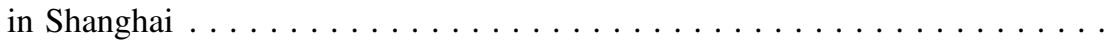


Design for Sustainable Behaviour

Xu Sun, Qingfeng Wang, Nan Wang, Charlie Sugianto So, and Yan Wang

Preliminary Study: Influence of Cultural Differences on the Innovation Process Between Chinese and Germans . Liuxing Tsao, Philip Alexander Behr-Heyder, and Liang Ma

From Invisible to Visible: The Evolution of Space Formation of the Nineteenth Century Christian Missionary Work in Taiwan ........ Yin-Chun Tseng, Kun-Chen Chang, Fu-Kuo Mii, and Chiu-Wei Chien

Exploring Socioeconomic and Sociocultural Implications of ICT Use:

An Ethnographic Study of Indigenous People in Malaysia . 403 Norazlinawati Walid, Emma Nuraihan Mior Ibrahim, Chee Siang Ang, and Norlaila Md. Noor

Stakeholder Engagement: Applying Dechnology in a Technology-Oriented Organization.

Chih-Shiang (Mike) Wu, William Huang, Pei-Lin Chen, and Tung-Jung Sung 